\title{
-Original-
}

\section{Human apolipoprotein B transgenic SHR/NDmcr-cp rats show exacerbated kidney dysfunction}

\author{
Makoto ASAHINA, Fumi SHIMIZU, Masayuki OHTA, Michiyasu TAKEYAMA, and \\ Ryuichi TOZAWA
}

Pharmaceutical Research Division, Takeda Pharmaceutical Company Ltd., 26-1, Muraoka-Higashi 2chome, Fujisawa, Kanagawa 251-8555, Japan

\begin{abstract}
Nephropathy frequently co-occurs with metabolic syndrome in humans. Metabolic syndrome is a cluster of metabolic diseases including obesity, diabetes, hypertension, and dyslipidemia, and some previous studies revealed that dyslipidemia contributes to the progression of kidney dysfunction. To establish a new nephropathy model with metabolic syndrome, we produced human apolipoprotein B (apoB) transgenic (Tg.) SHR/NDmcr-cp (SHR-cp/cp) rats, in which dyslipidemia is exacerbated more than in an established metabolic syndrome model, SHR-cp/cp rats. Human apoB Tg. SHR-cp/ $c p$ rats showed obesity, hyperinsulinemia, hypertension, and severe hyperlipidemia. They also exhibited exacerbated early-onset proteinuria, accompanied by increased kidney injury and increased oxidative and inflammatory markers. Histological analyses revealed the characteristic features of human apoB Tg. SHR-cp/cp rats including prominent glomerulosclerosis with lipid accumulation. Our newly established human apoB Tg. SHR-cp/cp rat could be a useful model for the nephropathy in metabolic syndrome and for understanding the interaction between dyslipidemia and renal dysfunction in metabolic syndrome.
\end{abstract}

Key words: apolipoprotein B, dyslipidemia, kidney dysfunction, metabolic syndrome, SHR/NDmcr-cp rats

\section{Introduction}

Metabolic syndrome is a cluster of metabolic diseases including obesity, diabetes, hypertension, and dyslipidemia, which greatly promotes the progression of renal dysfunction $[3,10]$. Metabolic syndrome and nephropathy are frequent complications in humans $[3,10]$. Recent clinical studies have revealed that metabolic syndrome increases the risk for chronic kidney disease (CKD) $[3,10]$.

SHR/NDmcr- $c p$ (SHR- $c p / c p$ ) is a rat model of metabolic syndrome with hypertension, obesity, hyperinsulinemia, and mild hyperlipidemia [4, 22]. SHR-cp/cp rats also spontaneously develop abnormalities in renal histology [4].

Therefore, we produced a new nephropathy model by provoking severe hyperlipidemia in SHR- $c p / c p$ rats. Dyslipidemia in type 2 diabetes patients is a risk factor for the progression of kidney dysfunction [16, 23]. Dyslipidemia is also considered to be associated with an increased rate of progression of renal insufficiency in nondiabetic patients [17]. Several studies in animal models have shown that hypercholesterolemia accelerates the rate of progression of kidney disease $[1,5,12]$. Thus we intended to establish an animal model for the progressive loss of kidney function, exacerbated by hyperlipidemia.

(Received 2 January 2015 / Accepted 15 March 2015 / Published online in J-STAGE 20 April 2015)

Address corresponding: M. Asahina, Integrated Technology Research Laboratories, Pharmaceutical Research Division, Takeda Pharmaceutical Company Ltd., 26-1, Muraoka-Higashi 2chome, Fujisawa, Kanagawa 251-8555, Japan

Supplementary figure: refer to J-STAGE: https://www.jstage.jst.go.jp/browse/expanim

(C)2015 Japanese Association for Laboratory Animal Science 
To exacerbate dyslipidemia in SHR- $c p / c p$ rats, we generated human apolipoprotein B-100 (apoB-100) transgenic (Tg.) SHR-cp/cp rats. ApoB plays a central role in mammalian lipoprotein metabolism, and apoB100 is the key structural protein in the formation of lipoproteins [29]. Human apoB-100 Tg. mice show increased plasma total cholesterol and triglyceride levels as compared with non-Tg. mice $[2,11]$. It could be expected that a transgene of human apoB in rats would provoke hyperlipidemia due to increased production of human apoB in the liver and/or reduced catabolism of human apoB-containing lipoproteins, as well as in mice $[2,11]$. In fact, human LDL binds poorly to rat cell receptors on rat fibroblasts and smooth muscle cells [7].

In the present study, to establish a human apoB $\mathrm{Tg}$. SHR- $c p / c p$ rats as a hyperlipidemia-dependent nephropathy model with metabolic syndrome, metabolic and biological parameters in urine and plasma, and renal histopathology were investigated.

\section{Materials and Methods}

All animal care procedures and experiments conformed to the guidelines of the AAALAC and were approved by the Experimental Animal Care and Use Committee of Takeda Pharmaceutical Company Limited.

Establishment of human apoB-100 transgenic (Tg.) SHRcp/cp rats.

To generate human apoB-100 transgenic rats, we injected human genomic DNA containing the full length of the human apoB-100 gene into fertilized eggs of Wistar rats. To select a transgenic rat line showing stable hyperlipidemia, expression analyses and phenotypic analyses were carried out among the four lines obtained. Then the male rats carrying the human apoB-100 transgene were backcrossed four times with SHR-+/+ rats to replace the genome of the Wistar rat by the speed congenic method. A TaqMan Copy Number Assay (HS02237611_cn, Life Technologies, Carlsbad, CA, USA) was used to detect human apoB transgene in the genome of rats. Then male N4 rats were crossed with SHR $-c p /+$ rats to introduce $c p$ mutations into the N5 rats. Finally, backcrosses to SHR rats were carried out nine times. Then human apoB Tg. SHR-cp/+ rats were crossed with non-Tg. SHR $-c p /+$ rats to obtain human apoB Tg. SHR-cp/cp rats and their littermates. Selection of the best human apoB Tg. male rats for subsequent backcross- ing was based on genotyping by MAX-BAX service (Charles River) using 110 markers scattered throughout the whole genome. SHR- $c p / c p$, SHR- $c p /+$, and SHR-+/+ rats were provided by the Disease Model Cooperative Research Association (Kyoto, Japan). The human apoB Tg. SHR-cp/+ rats will be deposited in the National BioResource Project for the Rat in Japan and will be available from the project.

\section{Animals}

All rats were housed in individual metal cages in a room with controlled temperature $\left(23^{\circ} \mathrm{C}\right)$, humidity (55\%), and lighting (lights on from 7:00 am to 7:00 pm) and were fed MF-based western diet (Oriental Yeast) containing $0.15 \%$ cholesterol and $15 \%$ butter for 7 months beginning at 8 weeks of age, with free access to water.

\section{Biochemical parameters}

Blood samples were collected from the tail vein with EDTA as an anticoagulant. Plasma HDL was fractionated with a commercially available kit (Wako Pure Chemical Industries). Then the total and HDL cholesterol levels were determined by enzyme assay kits (Wako Pure Chemical Industries). A turbidimetric immunoassay kit for the quantitative measurement of human apoB-100 and apoB-48 was used (Apo B auto N Daiichi, Sekisui Medical, Tokyo, Japan). Plasma triglyceride (TG), glucose, and blood urea nitrogen (BUN) were also measured with enzyme assay kits (Wako Pure Chemical Industries). Plasma insulin and C-reactive protein (CRP) concentrations were determined with ELISA kits (Morinaga and Alpha Diagnostic Intl). Lipid peroxide levels were estimated by TBARS assay kits (Cayman Chemical Company). To collect urine samples, the rats were placed individually in metabolic cages for $24 \mathrm{~h}$, and urine was collected with free access to water as well as western diet. Urinary albumin and protein were measured by a commercially available kit (Wako Pure Chemical Industries). Urinary kidney injury molecule 1 (KIM1) levels were determined by KIM1 rat ELISA kits (Abcam, Cambridge, UK)

\section{$R T-P C R$}

Thirty-six-week-old rats were sacrificed, and total RNA in the renal cortex was extracted with ISOGEN kits (Nippon Gene, Tokyo, Japan). The RNA was treated with DNase I (QIAGEN, Venlo, Netherlands). Then first- 
Table 1. Metabolic parameters in human apoB Tg. SHR-cp/cp rats

\begin{tabular}{lcccc}
\hline & ApoB Tg. $c p / c p$ & Non-Tg. $c p / c p$ & ApoB Tg. $+/+$ & Non-Tg. $+/+$ \\
\hline $\mathrm{n}$ & 9 & 8 & 7 & 7 \\
Body weight $(\mathrm{g})$ & $911 \pm 20.8$ & $963 \pm 8.6$ & $520 \pm 15.5^{* *}$ & $541 \pm 11.9^{* *}$ \\
Plasma insulin (ng/ml) & $35 \pm 5.9$ & $23 \pm 1.6$ & $3 \pm 0.2^{* *}$ & $3 \pm 0.3^{* *}$ \\
Plasma glucose (mg/dl) & $202 \pm 33.8$ & $203 \pm 26.9$ & $129 \pm 2.7$ & $145 \pm 2.5$ \\
Plasma TC (mg/dl) & $1,513 \pm 89.1$ & $176 \pm 9.2^{* *}$ & $465 \pm 10.1^{* *}$ & $73 \pm 1.8^{* *}$ \\
Plasma TG (mg/dl) & $1,044 \pm 61.4$ & $313 \pm 149.9^{* *}$ & $361 \pm 12.8^{* *}$ & $82 \pm 10.5^{* *}$ \\
Plasma human apoB (mg/dl) & $1,147 \pm 40.2$ & $0 \pm 0^{* *}$ & $406 \pm 11.6^{* *}$ & $0 \pm 0^{* *}$ \\
Plasma HDL-c (mg/dl) & $18 \pm 2.1$ & $40 \pm 2.3^{* *}$ & $5 \pm 0.3^{* *}$ & $24 \pm 1.0$ \\
Systolic blood pressure (mmHg) & $139 \pm 9.0$ & $153 \pm 2.0$ & $150 \pm 3.0$ & $168 \pm 4^{* *}$ \\
\hline
\end{tabular}

Data are shown as the mean $\pm \mathrm{SE}$. $* * P<0.01$ compared with human apoB Tg.-cp/cp rats.

strand cDNA was synthesized using a PrimeScript RT reagent Kit (Takara Bio, Otsu, Japan). We carried out real-time quantitative RT-PCR for the mRNA expression of secreted phosphoprotein1 (Spp 1) in the renal cortex by using a 7900HT Fast Real-Time PCR System with commercial TaqMan probe kits (TaqMan Gene Expression Assays, (Life Technologies) and TaqMan Universal PCR Master Mix (Life Technologies) according to a standard method. Actb was used as a housekeeping reference gene.

\section{Histopathology}

Kidneys were collected from human apoB Tg. SHR$c p / c p$ rats, non-Tg. SHR-cp/cp rats, human apoB Tg. SHR-+/+ rats, and non-Tg. SHR-+/+ rats. Then they were fixed in $10 \mathrm{vol} \%$ neutral buffered formalin and embedded in paraffin. The sections were stained with hematoxylin and eosin (H\&E) and periodic acid-methenamine silver (PAM). Frozen sections of the kidney from apoB Tg. SHR- $c p / c p$ rats and non-Tg. SHR- $c p / c p$ rat kidneys were also stained with Oil Red O. Using light microscopy, the severities of the tubulointerstitial findings such as degeneration/regeneration, mononuclear cell infiltration, and hyaline casts were determined in H\&E-stained sections according to the following criteria expressed on an arbitrary scale from 0 to 4 or 3: tubular degeneration/ regeneration $(0$, no tubular degeneration and regeneration; 1 , focal lesions; 2 , almost $25 \%$ of tubules were affectedd; 3 , almost $50 \%$ of tubules were affected; 4 , $>80 \%$ of tubules were affected), mononuclear cell infiltration $(0$, no mononuclear cell infiltration; 1 , sporadic and focal infiltrations; 2 , multifocal infiltrations; 3 , more extensive multifocal infiltrations), and hyaline casts ( 0 , no hyaline cast; 1 , a few hyaline casts; 2 , multiple hyaline casts mainly in the medulla; 3 , multiple hyaline casts in the medulla and sporadic hyaline casts in the cortex;
4 , numerous hyaline casts). The severity of the glomerulosclerosis was determined in the PAM-stained sections according to the following criteria expressed on an arbitrary scale from 0 to 4: 0 , no glomerulosclerosis; 1 , sporadic glomerulosclerosis; 2 , sporadic glomerulosclerosis, with adhesion to the Bowman's capsule in some affected glomeruli; 3 , sclerosis lesions in almost $50 \%$ of glomeruli, with adhesion to the Bowman's capsule in less than $50 \%$ of the affected glomeruli; 4 , sclerosis lesions in most glomeruli, with adhesion to the Bowman's capsule in more than $50 \%$ of the affected glomeruli. In addition, lipid accumulation in the glomerulus was evaluated using sections stained with Oil Red O.

\section{Statistical analysis}

Results were expressed as the mean \pm standard error. Statistical significance among four groups was determined using one-way ANOVA and subsequent parametric Dunnett's or Tukey's multiple comparison tests. Statistical significance between two strains was determined by Student's $t$-test.

\section{Results}

\section{Metabolic parameters}

Metabolic parameters were measured to confirm whether human apoB Tg. SHR-cp/cp rats met the criterion for metabolic syndrome. Thirty-six-week-old male human apoB Tg. SHR-cp/cp rats showed higher body weight levels when fed the western diet than human apoB Tg. SHR-+/+ and non-Tg. SHR-+/+ rats (Table 1). There were no significant differences in body weight between human apoB Tg. SHR- $c p / c p$ rats and non-Tg. SHR-cp/ $c p$ rats (Table 1). Twelve-week-old male human apoB $\mathrm{Tg}$. SHR- $c p / c p$ rats showed higher plasma insulin levels when fed the western diet than human apoB Tg. SHR-+/+ 
and non-Tg. SHR-+/+ rats after overnight fasting, although there were no significant differences in plasma glucose levels between the 4 strains (Table 1). There were no significant differences in plasma insulin levels between human apoB Tg. SHR-cp/cp rats and non-Tg. SHR-cp/cp rats (Table 1). Twelve-week-old human apoB Tg. SHR-cp/cp rats showed plasma total cholesterol (TC) and TG levels of more than $1,000 \mathrm{mg} / \mathrm{dl}$ when fed the MF-based western diet beginning at 8 weeks old. Then human apoB Tg. SHR-cp/cp rats showed stable hyperlipidemia during the experimental period (Supplemental Fig. 1). Thirty-six-week-old male human apoB Tg. SHR$c p / c p$ rats also showed higher total plasma TC, TG, and human apoB levels when fed the western diet than their littermates, human apoB Tg. SHR-+/+, non-Tg. SHR-cp/ $c p$, and non-Tg. SHR-+/+ rats (Table 1). Plasma human apoB protein was detected in only human apoB $\mathrm{Tg}$. rats (Table 1). Thirty-six-week-old male human apoB Tg. SHR- $c p / c p$ rats showed lower plasma HDL-cholesterol levels when fed the western diet than non-Tg. SHR-cp/ $c p$ rats (Table 1). Systolic blood pressure (SBP) in each strain was measured by the tail-cuff method in 12-weekold rats, and there were no significant differences among human apoB Tg. SHR-cp/cp, human apoB Tg. SHR-+/+, and non-Tg. SHR-cp/cp rats (Table 1). However, non-Tg. SHR-+/+ rats showed a significantly higher SBP than human apoB Tg. SHR-cp/cp rats. The human apoB transgene had no effect on metabolic parameters, obesity, diabetes, or hypertension, with the exception of hyperlipidemia.

Urinary albumin excretion, urinary protein excretion, and $B U N$

Urinary albumin and protein excretion (UAE and UPE) and BUN were measured in 12-, 20-, 28- and 36-week-old rats. UAE and UPE in male human apoB Tg. SHR- $c p / c p$ rats were more prominent earlier than in non-Tg. SHR- $c p / c p$ rats; when they were fed the western diet (Figs. 1A and 1B). BUN gradually increased in human apoB Tg. SHR-cp/cp rats in accordance with increases in UAE and UPE, but this did not occur in the non-Tg. SHR- $c p / c p$ rats (Fig. 1C).

\section{KIM1, Spp1, TBARS, and CRP}

Kidney injury markers, an oxidative stress marker, and inflammation markers were measured in 36-weekold rats. KIM1 is located in the renal proximal tubule epithelial cells, and urinary KIM1 is a specific biomark-
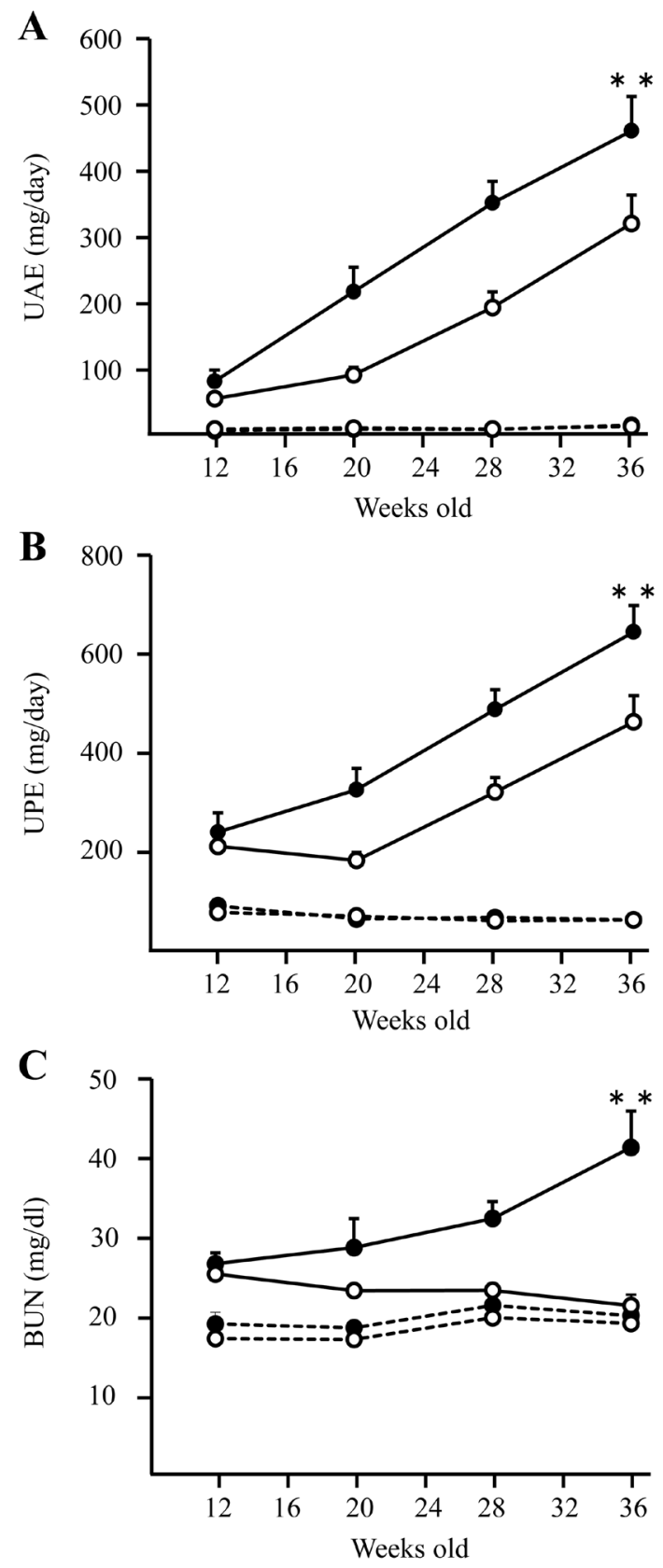

Fig. 1. (A)Urinary albumin excretion (UAE), (B) urinary protein excretion (UPE), and (C) BUN of rats. Filled circles and solid line, human apoB Tg. SHR-cp/cp $(\mathrm{n}=9)$; open circles and solid line, non-Tg. SHR- $c p / c p(\mathrm{n}=8)$; filled circles and dashed line, human apoB Tg. SHR- $+/+(n=7)$; and open circles and dashed line, non-Tg. SHR- $+/+$ rats $(n=7)$. The asterisks indicate a significant difference at $* * P<0.01$ in comparison of human apoB Tg. SHR-cp/cp rats with nonTg. SHR- $c p / c p$ rats.

er for tubular injury. Thirty-six-week-old male human apoB Tg. SHR- $c p / c p$ rats showed higher urinary KIM1 levels when fed the western diet than an established 

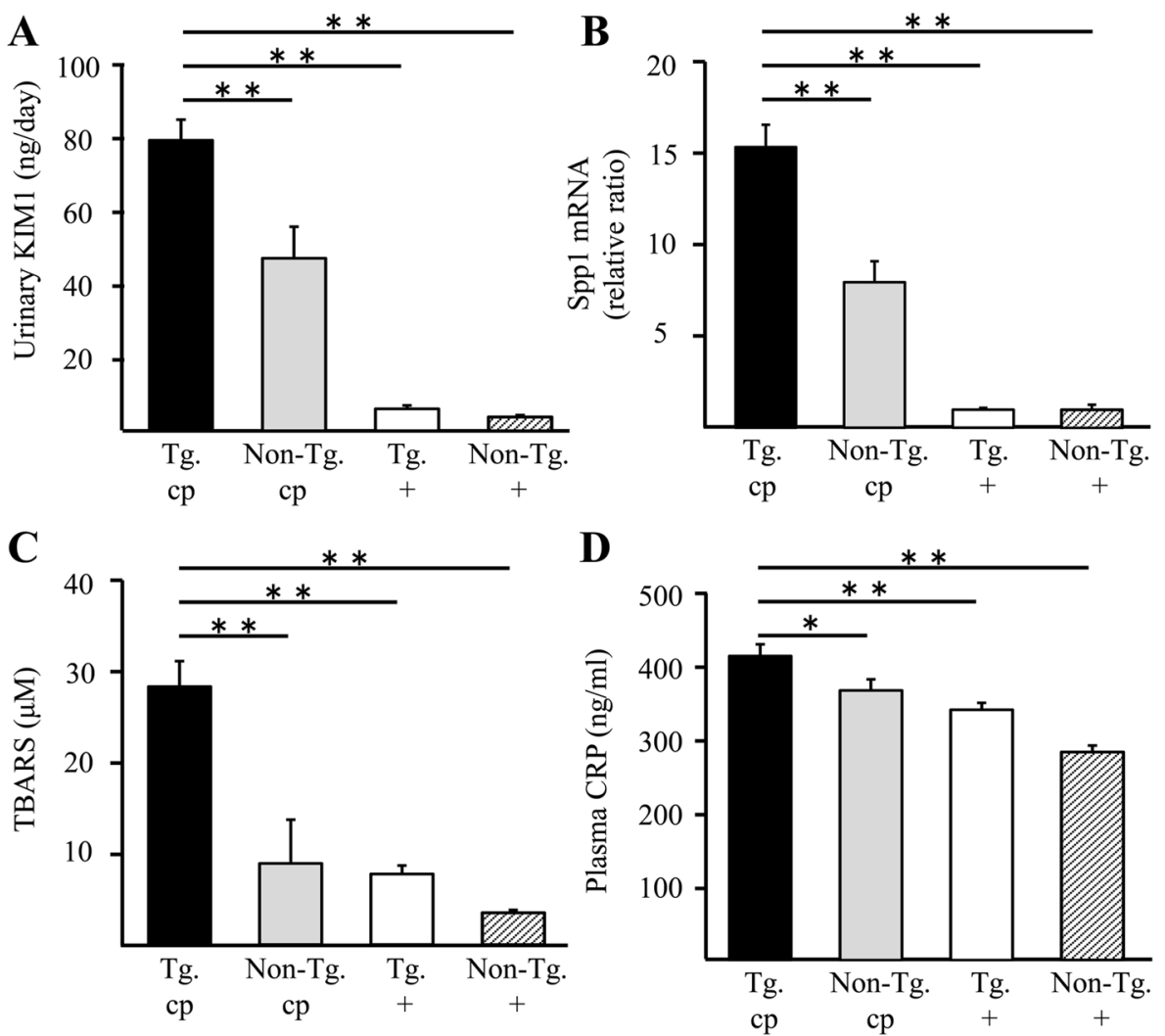

Fig. 2. Kidney injury, oxidative, and inflammation marker levels in 36-week-old of human apoB Tg. SHR- $c p / c p(\mathrm{n}=9)$, non-Tg. SHR- $c p / c p(\mathrm{n}=8)$, human apoB Tg. SHR- $+/+(\mathrm{n}=7)$, and nonTg. SHR-+/+ rats (n=7). (A) Urinary KIM1 excretion, (B) Spp1 mRNA expression in the kidney cortex, (C) plasma thiobarbituric acid reactive substances (TBARS), and (D) plasma $\mathrm{C}$-reactive protein (CRP) levels. The asterisks indicate a significant difference at $* P<0.05$ or $* * P<0.01$ in comparison of human apoB Tg. SHR- $c p / c p$ rats with their littermates.

diabetic nephropathy model, non-Tg. SHR-cp/cp rats (Fig. 2A). SPP1 is a pleiotropic cytokine that is ubiquitously expressed and upregulated during inflammation. Thirty-six-week-old male human apoB Tg. SHR-cp/cp rats also showed increased Spp1 mRNA expression levels in the renal cortex; compared with non-Tg. SHR-cp/ $c p$ rats (Fig. 2B). The plasma levels of TBARS, an oxidative stress marker and an index for lipid peroxidation are shown in Fig. 2C. Thirty-six-week-old male human apoB Tg. SHR-cp/cp rats showed more than a three-fold increase in TBARS levels than their littermates, human apoB Tg. SHR- $+/+$, non-Tg. SHR-cp/cp, and non-Tg. SHR-+/+ rats (Fig. 2C). The plasma levels of CRP, a sensitive marker for inflammation, are shown in Fig. 2D. Thirty-six-week-old male human apoB Tg. SHR-cp/cp rats showed significantly increased plasma hsCRP levels than those of their littermates, human apoB Tg. SHR-+/+, non-Tg. SHR- $c p / c p$, and non-Tg. SHR-+/+ rats (Fig. 2D).

\section{Renal histology}

Representative histological features of 36-week-old male human apoB Tg. SHR-cp/cp rats are shown in Fig. 3. Human apoB Tg. SHR-cp/cp rats showed prominent hyaline casts and tubular degeneration/regeneration. The tubular changes were characterized by vacuolation and single-cell necrosis of the tubular epithelium, thickening of the basement membrane and tubular dilation. Mononuclear cell infiltration mainly consisting of lymphocytes was also observed in the interstitial region (Fig. 3A). These lesions were also seen in non-Tg. SHR$c p / c p$ rats (Fig. $3 \mathrm{~B}$ ); however, the severities in human apoB Tg. SHR- $c p / c p$ rats were significantly higher than those in non-Tg. SHR-cp/cp rats (Table 2). Human apoB $\mathrm{Tg}$. SHR- $c p / c p$ rats also showed more severe glomerulosclerosis compared with non-Tg. SHR- $c p / c p$ rats (Figs. 3E and 3F), and the glomerulosclerosis score in human apoB Tg. SHR-cp/cp rats was significantly high- 

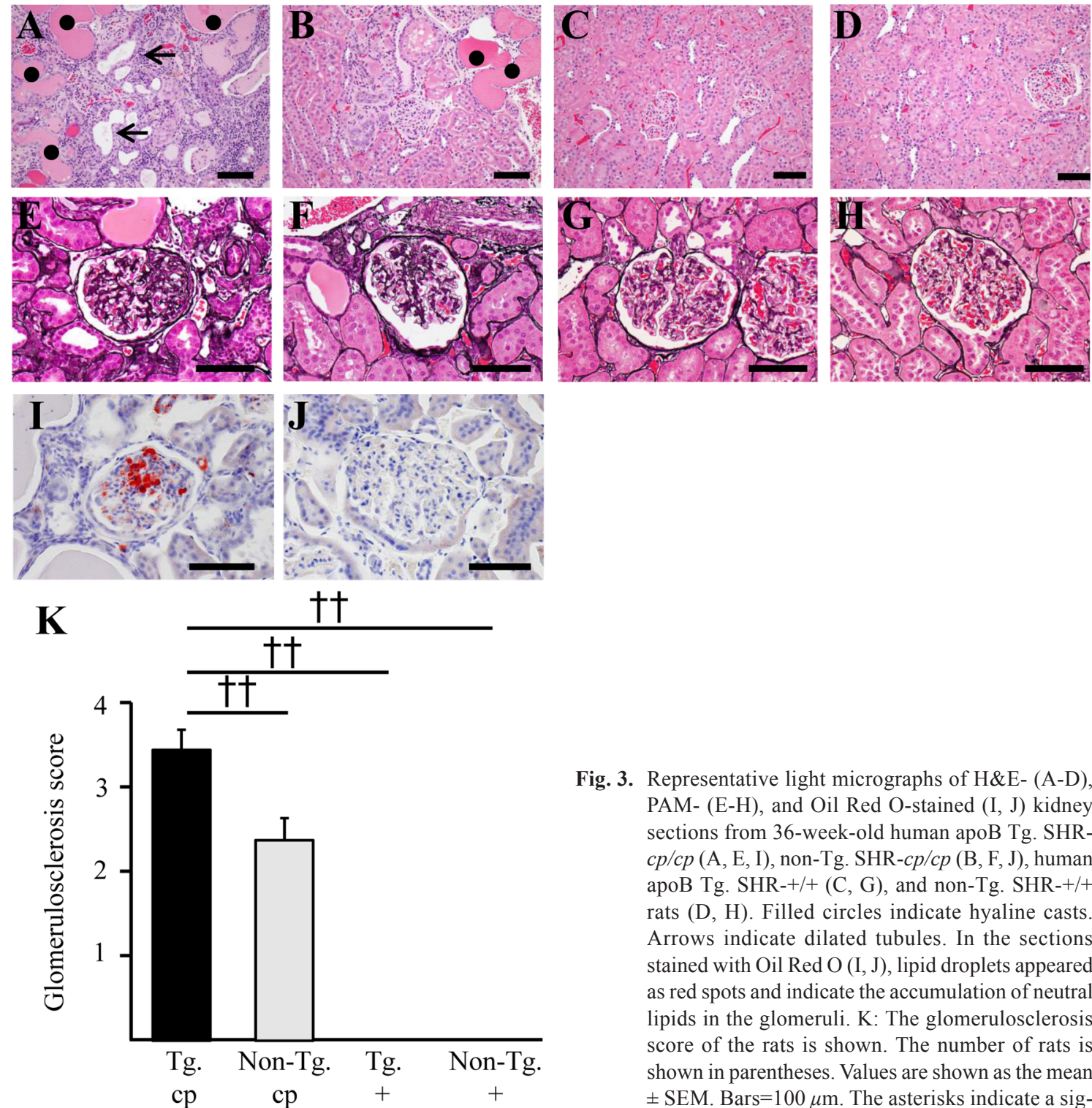

Fig. 3. Representative light micrographs of H\&E- (A-D), PAM- (E-H), and Oil Red O-stained (I, J) kidney sections from 36-week-old human apoB Tg. SHR$c p / c p(\mathrm{~A}, \mathrm{E}, \mathrm{I})$, non-Tg. SHR-cp/cp (B, F, J), human apoB Tg. SHR-+/+ (C, G), and non-Tg. SHR-+/+ rats $(\mathrm{D}, \mathrm{H})$. Filled circles indicate hyaline casts. Arrows indicate dilated tubules. In the sections stained with Oil Red O (I, J), lipid droplets appeared as red spots and indicate the accumulation of neutral lipids in the glomeruli. K: The glomerulosclerosis score of the rats is shown. The number of rats is shown in parentheses. Values are shown as the mean \pm SEM. Bars $=100 \mu \mathrm{m}$. The asterisks indicate a significant difference at ${ }^{\dagger \dagger} P<0.01$ compared with the human apoB Tg. SHR-cp/cp rats.

Table 2. Quantitative analysis of tubulointerstitial lesions in human apoB Tg. SHR-cp/cp rats

\begin{tabular}{lcccc}
\hline & ApoB Tg. $c p / c p$ & Non-Tg. $c p / c p$ & ApoB Tg. $+/+$ & Non-Tg. $+/+$ \\
\hline $\mathrm{n}$ & 9 & 8 & 7 & 7 \\
Tubular degeneration/regeneration & $2.9 \pm 0.1$ & $2.1 \pm 0.1^{*}$ & $0.7 \pm 0.2^{* *}$ & $0.6 \pm 0.2^{* *}$ \\
Mononuclear cell infiltration & $2.8 \pm 0.2$ & $1.8 \pm 0.2^{*}$ & $0.1 \pm 0.2^{* *}$ & $0.1 \pm 0.2^{* *}$ \\
Hyaline cast & $2.9 \pm 0.1$ & $2.3 \pm 0.2^{*}$ & $0.9 \pm 0.2^{* *}$ & $0.7 \pm 0.2^{* *}$ \\
\hline
\end{tabular}

Data are shown as the mean \pm SE. $* P<0.05$ and $* * P<0.01$ compared with human apoB Tg. $-c p / c p$ rats.

er than that in non-Tg. SHR-cp/cp rats (Fig. 3K). Few lipids were observed in the glomeruli of non-Tg. SHR$c p / c p$ rats (Fig. $3 \mathrm{~J}$ ), whereas more prominent lipid accumulation was observed in the glomeruli of all the human apoB Tg. SHR-cp/cp rats (Fig. 3I). Human apoB Tg. SHR-+/+ rats and non-Tg. SHR-+/+ rats showed no remarkable glomerular or tubulointerstitial changes (Figs. 3C, 3D, 3G, and 3H). 


\section{Discussion}

In this study, we established a new nephropathy model with metabolic syndrome, human apoB Tg. SHR-cp/ $c p$ rats, by provoking severe hyperlipidemia. Human apoB Tg. SHR- $c p / c p$ rats showed early-onset proteinuria and exacerbated kidney dysfunction compared with an established model, SHR-cp/cp rats.

Glomerular and tubulointerstitial lesions were more pronounced in the kidneys of human apoB Tg. SHR-cp/ $c p$ rats, compared with non-Tg. SHR- $c p / c p$ rats. Human apoB Tg. SHR- $c p / c p$ rats showed marked glomerulosclerosis. Glomerulosclerosis is a hallmark of diabetic nephropathy. They also showed lipid accumulation in the glomeruli. Several studies have shown the presence of lipid accumulation in the kidneys of diabetic patients [6] and animal models $[8,12,20,26]$. It has been suggested that lipid accumulation in the kidney could play a role in the pathogenesis of diabetic nephropathy $[6,8,12,20,26]$. It has been thought that lipid accumulation in glomeruli and subsequent lipotoxicity would cause podocyte dysfunction and apoptosis [6], activate inflammatory mediators [9], and finally cause kidney injury, although the detailed pathogenic process in human apoB Tg. SHR-cp/cp rats has not been clarified.

Thirty-six-week-old male human apoB Tg. SHR-cp/ $c p$ rats showed significantly increased urinary KIM1 levels compared with non-Tg. SHR-cp/cp rats. KIM1 is a type I transmembrane structural glycoprotein located in the renal proximal tubular epithelia. These cells undergo regeneration after various forms of injury and shed KIM1 antigen into the urine. Thus urinary KIM1 could be a specific biomarker for renal tubular injury. Actually, increased urinary KIM1 is an independent predictor of long-term renal graft loss and is also elevated in human proteinuric CKD [24, 25]. Male human apoB Tg. SHR-cp/cp rats also showed significantly increased Sppl mRNA expression levels in the renal cortex, compared with non-Tg. SHR- $c p / c p$ rats. SPP1 is a pleiotropic cytokine that is broadly expressed and upregulated during inflammation. Gene expression studies showed that higher Spp1 levels correlate with more severe diabetic albuminuria and glomerulosclerosis in diabetic nephropathy models [21]. Spp1 expression is induced in several models of tubulointerstitial disease [27]. In addition, inducible expression of Sppl in the tubular epithelium seems to be associated with interstitial monocyte infiltration and subsequent tubulointerstitial changes in some forms of human renal disease [15]. These changes in injury markers in human apoB Tg. SHR-cp/cp rats were consistent with histological changes of glomeruli and tubulointerstitial area in human apoB Tg. SHR-cp/cp rats.

Plasma hydroperoxide levels were measured to evaluate the degree of lipid peroxidation of blood components. We found that TBARS, an index for lipid peroxidation, increased in the plasma of human apoB $\mathrm{Tg}$. SHR-cp/cp rats. The extremely high cholesterol and TG levels contribute to the marked elevations of circulating hydroperoxide in rats $[18,28]$. Thus it could be suggested that severe hyperlipidemia causes lipid peroxidation, an oxidative stress marker in human apoB $\mathrm{Tg}$. SHR- $c p / c p$ rats.

Human apoB Tg. SHR-cp/cp rats also showed significantly increased plasma CRP levels compared with their littermates, human apoB Tg. SHR-+/+, non-Tg. SHR- $c p / c p$, and non-Tg. SHR-+/+ rats. These results suggested that human apoB Tg. SHR-cp/cp rats have chronic middle-grade inflammation [28]. In a previous study, it was demonstrated that elevated serum CRP levels were positively associated with renal function abnormality and that early inflammatory processes may predispose the kidney to glomerular hyperfiltration-related renal function loss in humans [19]. It has been suggested that severe hyperlipidemia induced by the human apoB transgene in SHR-cp/cp rats could influence the inflammation and oxidative stress, although the human apoB transgene had no effect on metabolic parameters including obesity, diabetes, and hypertension. Yu Yamaguchi et al. confirmed that serum oxidative stress markers notably increase in SHR-cp/cp rats [28]. Miki Nagase et al. suggested that oxidative stress caused by mineralocorticoid receptor activation would partly contribute to this renal abnormality in SHR-cp/cp rats [13]. These results suggested that oxidative stress could partly be involved in the renal injury in human apoB Tg. SHR-cp/cp rats characterized by glomerular sclerosis. Actually, biomarkers of inflammation and oxidative stress status are increased in chronic kidney dysfunction patients compared with healthy subjects [14]. Increased prevalence of oxidative stress and inflammation could also contribute to the progression of kidney dysfunction [14].

In the present study, we produced human apoB $\mathrm{Tg}$. SHR- $c p / c p$ rats to introduce the human apoB-100 gene into SHR- $c p / c p$ rats, a metabolic syndrome model. How- 
ever, the human apoB transgene had no effect on metabolic parameters, obesity, diabetes, or hypertension among rats with $c p$ mutations, although inflammation and oxidative stress status were increased in human apoB $\mathrm{Tg}$. SHR- $c p / c p$ rats; compared with non-Tg. SHR-cp/cp rats. It is suggested that the levels of inflammation and oxidative stress in human apoB Tg. SHR-cp/cp rats would be insufficient for exacerbating metabolic parameters.

The plasma lipids levels of human apoB Tg. SHR-cp/ $c p$ rats were three times higher than those of human apoB Tg. SHR-+/+ rats. However, the aortic atherosclerotic lesions were comparable between human apoB Tg. SHR$c p / c p$ rats and human apoB $\mathrm{Tg}$. SHR-+/+ rats (data not shown). Human apoB Tg. SHR-cp/cp rats also did not show prominent atherosclerotic plaques and coarctation of renal arteries. Thus it is suggested that the aortic lipid deposition would not cause exacerbated kidney dysfunction in human apoB Tg. SHR-cp/cp rats.

In summary, human apoB Tg. SHR-cp/cp rats developed progressive kidney failure, exacerbated by hyperlipidemia with metabolic syndrome. Our newly established metabolic syndrome model, the human apoB Tg. SHR- $c p / c p$ rat, is a useful model for understanding the interaction between hyperlipidemia and renal failure. It would be possible to use this model to develop therapeutic agents for kidney dysfunction associated with metabolic syndrome. Further study will be necessary to determine the involvement of lipotoxicity and oxidative stress in the pathogenesis and pathology of this metabolic syndrome model.

\section{Acknowledgments}

We thank Takeshi Yamamura, Kunio Matsuoka, Ritsuko Takami, and Hitoshi Kandori for expert technical help and Tadatoshi Hashimoto and Hirokazu Matsumoto for discussion.

\section{References}

1. Abrass, C.K. 2004. Cellular lipid metabolism and the role of lipids in progressive renal disease. Am. J. Nephrol. 24: 46-53. [Medline] [CrossRef]

2. Callow, M.J., Stoltzfus, L.J., Lawn, R.M., and Rubin, E.M. 1994. Expression of human apolipoprotein B and assembly of lipoprotein(a) in transgenic mice. Proc. Natl. Acad. Sci. USA 91: 2130-2134. [Medline] [CrossRef]

3. Chen, J., Muntner, P., Hamm, L.L., Jones, D.W., Batuman,
V., Fonseca, V., Whelton, P.K., and He, J. 2004. The metabolic syndrome and chronic kidney disease in U.S. adults. Ann. Intern. Med. 140: 167-174. [Medline] [CrossRef]

4. Ernsberger, P., Koletsky, R.J., and Friedman, J.E. 1999. Molecular pathology in the obese spontaneous hypertensive Koletsky rat: a model of syndrome X. Ann. N. Y. Acad. Sci. 892: 272-288. [Medline] [CrossRef]

5. Guijarro, C., Kasiske, B.L., Kim, Y., O’Donnell, M.P., Lee, H.S., and Keane, W.F. 1995. Early glomerular changes in rats with dietary-induced hypercholesterolemia. Am. J. Kidney Dis. 26: 152-161. [Medline] [CrossRef]

6. Herman-Edelstein, M., Scherzer, P., Tobar, A., Levi, M., and Gafter, U. 2014. Altered renal lipid metabolism and renal lipid accumulation in human diabetic nephropathy. J. Lipid Res. 55: 561-572. [Medline] [CrossRef]

7. Innerarity, T.L., Pitas, R.E., and Mahley, R.W. 1980. Disparities in the interaction of rat and human lipoproteins with cultured rat fibroblasts and smooth muscle cells. Requirements for homology for receptor binding activity. J. Biol. Chem. 255: 11163-11172. [Medline]

8. Jiang, T., Wang, Z., Proctor, G., Moskowitz, S., Liebman, S.E., Rogers, T., Lucia, M.S., Li, J., and Levi, M. 2005. Diet-induced obesity in C57BL/6J mice causes increased renal lipid accumulation and glomerulosclerosis via a sterol regulatory element-binding protein-1c-dependent pathway. J. Biol. Chem. 280: 32317-32325. [Medline] [CrossRef]

9. Kiss, E., Kränzlin, B., Wagenblaß, K., Bonrouhi, M., Thiery, J., Gröne, E., Nordström, V., Teupser, D., Gretz, N., Malle, E., and Gröne, H.J. 2013. Lipid droplet accumulation is associated with an increase in hyperglycemia-induced renal damage: prevention by liver X receptors. Am. J. Pathol. 182: 727-741. [Medline] [CrossRef]

10. Kurella, M., Lo, J.C., and Chertow, G.M. 2005. Metabolic syndrome and the risk for chronic kidney disease among nondiabetic adults. J. Am. Soc. Nephrol. 16: 2134-2140. [Medline] [CrossRef]

11. Linton, M.F., Farese, R.V. Jr., Chiesa, G., Grass, D.S., Chin, P., Hammer, R.E., Hobbs, H.H., and Young, S.G. 1993. Transgenic mice expressing high plasma concentrations of human apolipoprotein B100 and lipoprotein(a). J. Clin. Invest. 92: 3029-3037. [Medline] [CrossRef]

12. Mazzolai, L., Korber, M., Bouzourene, K., Aubert, J.F., Nussberger, J., Stamenkovic, I., and Hayoz, D. 2006. Severe hyperlipidemia causes impaired renin-angiotensin system function in apolipoprotein E deficient mice. Atherosclerosis 186: 86-91. [Medline] [CrossRef]

13. Nagase, M., Matsui, H., Shibata, S., Gotoda, T., and Fujita, T. 2007. Salt-induced nephropathy in obese spontaneously hypertensive rats via paradoxical activation of the mineralocorticoid receptor: role of oxidative stress. Hypertension 50: 877-883. [Medline] [CrossRef]

14. Oberg, B.P., McMenamin, E., Lucas, F.L., McMonagle, E., Morrow, J., Ikizler, T.A., and Himmelfarb, J. 2004. Increased prevalence of oxidant stress and inflammation in patients with moderate to severe chronic kidney disease. Kidney Int. 65: 1009-1016. [Medline] [CrossRef]

15. Okada, H., Moriwaki, K., Konishi, K., Kobayashi, T., Sugahara, S., Nakamoto, H., Saruta, T., and Suzuki, H. 2000. Tu- 
bular osteopontin expression in human glomerulonephritis and renal vasculitis. Am. J. Kidney Dis. 36: 498-506. [Medline] [CrossRef]

16. Rutledge, J.C., Ng, K.F., Aung, H.H., and Wilson, D.W. 2010. Role of triglyceride-rich lipoproteins in diabetic nephropathy. Nat. Rev. Nephrol. 6: 361-370. [Medline] [CrossRef]

17. Samuelsson, O., Mulec, H., Knight-Gibson, C., Attman, P.O., Kron, B., Larsson, R., Weiss, L., Wedel, H., and Alaupovic, P. 1997. Lipoprotein abnormalities are associated with increased rate of progression of human chronic renal insufficiency. Nephrol. Dial. Transplant. 12: 1908-1915. [Medline] [CrossRef]

18. Staprans, I., Rapp, J.H., Pan, X.M., and Feingold, K.R. 1993. The effect of oxidized lipids in the diet on serum lipoprotein peroxides in control and diabetic rats. J. Clin. Invest. 92: 638-643. [Medline] [CrossRef]

19. Stuveling, E.M., Hillege, H.L., Bakker, S.J., Gans, R.O., De Jong, P.E., and De Zeeuw, D. 2003. C-reactive protein is associated with renal function abnormalities in a non-diabetic population. Kidney Int. 63: 654-661. [Medline] [CrossRef]

20. Sun, L., Halaihel, N., Zhang, W., Rogers, T., and Levi, M. 2002. Role of sterol regulatory element-binding protein 1 in regulation of renal lipid metabolism and glomerulosclerosis in diabetes mellitus. J. Biol. Chem. 277: 18919-18927. [Medline] [CrossRef]

21. Susztak, K., Böttinger, E., Novetsky, A., Liang, D., Zhu, Y., Ciccone, E., Wu, D., Dunn, S., McCue, P., and Sharma, K. 2004. Molecular profiling of diabetic mouse kidney reveals novel genes linked to glomerular disease. Diabetes 53: 784794. [Medline] [CrossRef]

22. Takaya, K., Ogawa, Y., Hiraoka, J., Hosoda, K., Yamori, Y., Nakao, K., and Koletsky, R.J. 1996. Nonsense mutation of leptin receptor in the obese spontaneously hypertensive Koletsky rat. Nat. Genet. 14: 130-131. [Medline] [CrossRef]
23. Trevisan, R., Dodesini, A.R., and Lepore, G. 2006. Lipids and renal disease. J. Am. Soc. Nephrol. 17:(Suppl 2): S145S147. [Medline] [CrossRef]

24. van Timmeren, M.M., Vaidya, V.S., van Ree, R.M., Oterdoom, L.H., de Vries, A.P., Gans, R.O., van Goor, H., Stegeman, C.A., Bonventre, J.V., and Bakker, S.J. 2007. High urinary excretion of kidney injury molecule- 1 is an independent predictor of graft loss in renal transplant recipients. Transplantation 84: 1625-1630. [Medline] [CrossRef]

25. Waanders, F., Vaidya, V.S., van Goor, H., Leuvenink, H., Damman, K., Hamming, I., Bonventre, J.V., Vogt, L., and Navis, G. 2009. Effect of renin-angiotensin-aldosterone system inhibition, dietary sodium restriction, and/or diuretics on urinary kidney injury molecule 1 excretion in nondiabetic proteinuric kidney disease: a post hoc analysis of a randomized controlled trial. Am. J. Kidney Dis. 53: 16-25. [Medline] [CrossRef]

26. Wang, Z., Jiang, T., Li, J., Proctor, G., McManaman, J.L., Lucia, S., Chua, S., and Levi, M. 2005. Regulation of renal lipid metabolism, lipid accumulation, and glomerulosclerosis in FVBdb/db mice with type 2 diabetes. Diabetes 54: 2328-2335. [Medline] [CrossRef]

27. Xie, Y., Sakatsume, M., Nishi, S., Narita, I., Arakawa, M., and Gejyo, F. 2001. Expression, roles, receptors, and regulation of osteopontin in the kidney. Kidney Int. 60: 1645-1657. [Medline] [CrossRef]

28. Yamaguchi, Y., Yoshikawa, N., Kagota, S., Nakamura, K., Haginaka, J., and Kunitomo, M. 2006. Elevated circulating levels of markers of oxidative-nitrative stress and inflammation in a genetic rat model of metabolic syndrome. Nitric Oxide 15: 380-386. [Medline] [CrossRef]

29. Young, S.G. 1990. Recent progress in understanding apolipoprotein B. Circulation 82: 1574-1594. [Medline] [CrossRef] 